\title{
Mixed Problem for Hyperbolic Systems of First Order II
}

\author{
By \\ Masaru TANIGUCHI*
}

\section{Introduction}

We consider the mixed problems for the hyperbolic systems of first order

$$
\left\{\begin{array}{l}
\frac{\partial u}{\partial t}=A \frac{\partial u}{\partial x}+B \frac{\partial u}{\partial y}+f(t, x, y)=L[u]+f \\
u(0, x, y)=g(x, y) \\
K \cdot u(t, 0, y)=h(t, y) \quad t>0, x>0, y \in \mathbb{R}^{1},
\end{array}\right.
$$

where $u$ is an $N$-vector, $A$ and $B$ are $N \times N$ constant matrices, and $K$ is an $l \times N$ constant matrix with its rank $l$.

The aim of this paper is to obtain the energy inequalities of the solutions for the mixed problems (1.1). We prove this inequalities by the same method which Kajitani used in [4]. In Sec. 2, we explain notations. In Sec. 3, we construct the symbol of the singular integral operator which plays an essential role to prove the energy inequalities of the solution for the mixed problems with non-zero initial data. In Sec. 4, we prove the Main Theorem.

We assume the following conditions for the problem (1.1):

Condition I. The operator $\left(\frac{\partial}{\partial t}-L\right)$ is strongly hyperbolic, that is, for any $(\xi, \eta) \in \boldsymbol{R}^{2}-\{0\}, A \xi+B \eta$ has only real eigenvalues and is uni-

Communicated by S. Matsuura, September 21, 1973.

* Department of Mathematics, Faculty of Science and Technology, Science University of Tokyo, Tokyo.

Present Address: Department of Mathematics, Natural Sciences and Engineering Division, Ryukyu University, Tonokura-cho, Naha, Okinawa, 
formly diagonalizable in $(\xi, \eta)$.

Condition II. $A$ is non-singular.

Condition III. The boundary matrix $K$ satisfies the uniform Lopatinski's condition.

Remark 1. See [8] for another characterization of strong hyperbolicity.

We obtain the following:

Main Theorem. Assume the Conditions I, II and III. Then, there exist positive constants $C$ and $\mu_{0}$ such that, for any solution $u(t, x, y)$ of the problem (1.1) which belongs to $\mathscr{E}_{t}^{0}\left(H^{1}\left(\mathbb{R}_{+}^{2}\right)\right)$ and $\mathscr{E}_{t}^{1}\left(L^{2}\left(\boldsymbol{R}_{+}^{2}\right)\right)$

$$
\begin{gathered}
\left\|e^{-\mu t} u(t)\right\|^{2}+\int_{0}^{t} \mu\left\|e^{-\mu s} u(s)\right\|^{2}+<e^{-\mu s} u(s, 0, \cdot)>^{2} d s \\
\leqq C\left\{\|g\|^{2}+\int_{0}^{t} \frac{1}{\mu}\left\|e^{-\mu s} f(s)\right\|^{2}+<e^{-\mu s} h(s)>^{2} d s\right\}, \\
\text { for } t \geqq 0,{ }^{\forall} \mu \geqq \mu_{0} .
\end{gathered}
$$

(Notation is explained in Sec. 2.)

Remark 2. Strongly hyperbolic systems contain strictly and symmetric hyperbolic ones. Kajitani [4] and Rauch [6], [7] derived the $L^{2}$ estimates of solutions for the mixed problems with non-homogeneous conditions for strictly and symmetric hyperbolic systems. But nothing is known for strongly hyperbolic systems.

\section{Notation}

$$
\begin{array}{lll}
\boldsymbol{R}^{n}\left(\mathbb{C}^{n}\right) & : n \text {-dimensional real (complex) Euclidian space. } \\
\boldsymbol{R}_{+}^{n} & : \text { the set }\left\{(x, z) \mid x>0, z \in \boldsymbol{R}^{n-1}\right\} . \\
H^{s}\left(\boldsymbol{R}^{n}\right)\left(H^{s}\left(\boldsymbol{R}_{+}^{n}\right)\right): & \text { the space of functions whose partial derivatives } \\
& \text { of order } \leqq s \text { all exist and are square integrable } \\
& \text { in } \boldsymbol{R}^{n}\left(\boldsymbol{R}_{+}^{n}\right) . \\
\mathscr{E}_{t}^{k}(E) & : \text { the space of functions which are } k \text { times con- }
\end{array}
$$




$$
\begin{array}{ll} 
& \text { tinuously differentiable in } t \text { as } E \text {-valued function. } \\
\|\cdot\|(<\cdot>) \quad: \text { the norm of } L^{2}\left(\boldsymbol{R}_{+}^{2}\right)\left(L^{2}\left(\boldsymbol{R}^{1}\right)\right)
\end{array}
$$

\section{The Construction of a Symmetrizer}

Put $R(\xi, \eta)=A \xi+B \eta$. To prove the Main Theorem for strictly hyperbolic systems with $n$-space variables, Kajitani [4] used the singular integral operator whose symbol has the following properties:

For any real $(\xi, \eta) \neq(0,0)$

(i) $S(\xi, \eta)$ is a symmetric positive definite matrix with homogeneous degree zero and belongs to $C^{\infty}$ class.

(ii) $S(\xi, \eta) \cdot \widetilde{R}(\xi, \eta)$ is symmetric. $\left(\tilde{R}(\xi, \eta)=A \xi+\sum_{j=1}^{n-1} B_{j} \eta_{j}\right)$.

(iii) $S(\xi, 0)$ is a constant symmetric matrix.

(iv) $S(\zeta, \eta)$ is an analytic function of $\zeta$ in the strip

$$
|\operatorname{Im} \zeta| \leqq \delta|\eta|, \quad \eta \neq 0,
$$

where $\delta$ is a positive constant.

Since this singular integral operator is useful for the mixed problems (1.1), we shall prove that the singular integral operator whose symbol has the same properties, can be constructed for strongly hyperbolic systems with two space variables. We call such an operator symmetrizer [2].

We assume the condition I. Put $Q(\lambda, \xi, \eta)=\operatorname{det}(\lambda I-(A \xi+B \eta))$. Since the operator $\left(\frac{\partial}{\partial t}-L\right)$ is hyperbolic, the coefficients of the polynomial $Q(\lambda, \xi, \eta)$ in $(\lambda, \xi, \eta)$ are real. So, $Q(\lambda, \xi, \eta)$ has the following decomposition over the real field,

$$
Q(\lambda, \xi, \eta)=\prod_{i=1}^{H}\left[Q_{i}(\lambda, \xi, \eta)\right]^{h_{i}}
$$

where $Q_{i}(\lambda, \xi, \eta), \quad i=1, \ldots, H$ are irreducible homogeneous polynomials in $(\lambda, \xi, \eta)$ with degree $q_{i}$ and have the form

$$
Q_{i}(\lambda, \xi, \eta)=\lambda^{q_{i}}+a_{1}^{i}(\xi, \eta) \lambda^{q_{i}-1}+\cdots+a_{q_{i}}^{i}(\xi, \eta)
$$

and 


$$
\sum_{i=1}^{H} h_{i} q_{i}=N
$$

Since the roots of $\widetilde{Q}(\lambda, \xi, \eta)=0 \widetilde{Q}(\lambda, \xi, \eta)=\prod_{i=1}^{H} Q_{i}(\lambda, \xi, \eta)$ with respect to $\lambda$ are homogeneous of degree one in $(\xi, \eta)$, they are distinct on $S^{1}$ except for finite points by the theory of algebraic functions when we consider them on the unit sphere $S^{1}=\left\{(\xi, \eta) \in \boldsymbol{R}^{2} \mid \xi^{2}+\eta^{2}=1\right\}$. We denote the set of those points on $S^{1}$ by $E$. We have

$$
\begin{aligned}
Q_{i}(\lambda, \xi, \eta) & =\prod_{j=1}^{q_{i}}\left(\lambda-\theta_{j}^{i}(\xi, \eta)\right) \\
(\xi, \eta) & \in S^{1}-E, \quad(i=1, \ldots, H)
\end{aligned}
$$

where $\theta_{j}^{i} \neq \theta_{k}^{l}(i \neq l$ or $j \neq k$ ). After reordering and relabelling those roots in $S^{1}-E$, we obtain the equation

$$
Q(\lambda, \xi, \eta)=\prod_{j=1}^{M}\left(\lambda-\lambda_{j}(\xi, \eta)\right)^{m(j)}
$$

$$
(\xi, \eta) \in S^{1}-E
$$

where

$$
\left\{\begin{array}{l}
M=\sum_{i=1}^{H} q_{i} \\
N=\sum_{i=1}^{H} h_{i} q_{i}=\sum_{j=1}^{M} m(j)
\end{array}\right.
$$

and $m(j)=h_{i}$ if $\lambda_{j}(\xi, \eta)$ satisfies $Q_{i}(\lambda, \xi, \eta)=0$. According to the spectral theory of diagonalizable matrices, to $\lambda_{1}(\xi, \eta), \ldots, \lambda_{M}(\xi, \eta)$, there are corresponding matrices $P_{1}(\xi, \eta), \ldots, P_{M}(\xi, \eta)$, called eigenprojection and characterized by the conditions,

$$
\begin{array}{lc}
P_{j}(\xi, \eta) \text { has } \operatorname{rank} m(j) & (j=1, \ldots, M), \\
P_{i}(\xi, \eta) \cdot P_{j}(\xi, \eta)=\delta_{i j} P_{i}(\xi, \eta) & (i, j=1, \ldots, M), \\
\sum_{j=1}^{M} P_{j}(\xi, \eta)=I & \\
R(\xi, \eta) \cdot P_{j}(\xi, \eta)=P_{j}(\xi, \eta) \cdot R(\xi, \eta)=\lambda_{j}(\xi, \eta) \cdot P_{j}(\xi, \eta) \\
(\xi, \eta) \in S^{1}-E . & (j=1, \ldots, M)
\end{array}
$$


Lemma 3.1. (Taniguchi [9]) Assume the Condition I. Then we obtain:

(i) For any $(\xi, \eta) \in \boldsymbol{R}^{2}-\{0\}, R(\xi, \eta)=A \xi+B \eta$ has only real eigenvalues and is diagonalizable.

(ii) For any $(\xi, \eta) \in \boldsymbol{R}^{2}-\{0\}$, the eigenvalues and the associated eigenprojections of $R\left(w_{1}, w_{2}\right)=A w_{1}+B w_{2}$ are analytic in a neighborhood of $(\xi, \eta)$ in $\boldsymbol{C}^{2}$.

In [9], we applied the theory of algebraic functions to the equation $\operatorname{det}(\lambda I-(A+B \kappa))=0 \quad\left(\operatorname{det}\left(\lambda^{\prime} I-\left(A \kappa^{\prime}+B\right)\right)=0\right)$ with respect to $\lambda\left(\lambda^{\prime}\right)$ and derived the Lemma 3.1 by the fact that $\lambda\left(\lambda^{\prime}\right)$ is analytic (analytic) in $\kappa\left(\kappa^{\prime}\right)$ when $\kappa\left(\kappa^{\prime}\right)$ is real (real). By this method of proof and the homogenety of eigenvalues and associated eigenprojections, $\lambda_{j}(\xi, \eta)$ and $P_{j}(\xi$, $\eta), j=1, \ldots, M$, are distinct in a neighborhood of $\left(\xi_{*}, \eta_{*}\right)$ in $\mathbb{C}^{2}$ where $\left(\xi_{*}, \eta_{*}\right) \in S^{1}-E$, and are distinct in a neighborhood of $\left(\xi^{*}, \eta^{*}\right)$ in $\boldsymbol{C}^{2}$ except for the set $\left\{(\xi, \eta) \mid \xi^{*} \eta=\eta^{*} \xi\right\}$ where $\left(\xi^{*}, \eta^{*}\right) \in E$.

Definition 3.2. Let $F(x, y)$ be a multivalued analytic function of $(x, y)$ defined in a neighborhood of $S^{1}$ in $\mathbb{C}^{2}$ and $F_{r}(x, y)$ be its restriction on $S^{1}$. Then, we define that the period of $F(x, y)$ is the smallest number $\beta$ if the initial value of $F_{r}(x, y)$ agrees with its final one after $\beta$ times rotations with positive direction along $S^{1}$.

Put

$$
\Lambda(\xi, \eta)=\left\{\lambda_{1}(\xi, \eta), \ldots, \lambda_{M}(\xi, \eta)\right\}
$$

and

$$
\mathscr{P}(\xi, \eta)=\left\{P_{1}(\xi, \eta), \ldots, P_{M}(\xi, \eta)\right\}
$$

Lemma 3.3. Assume the Condition $I$. Then, the sets $\Lambda(\xi, \eta)$ and $\mathscr{P}(\xi, \eta)$ can be decomposed into disjoint sets of analytic functions defined in a neighborhood $S_{\boldsymbol{C}}^{1}$ of $S^{1}$ in $\boldsymbol{C}^{2}$,

$$
\begin{aligned}
& \Lambda_{j}(\xi, \eta)=\left\{\lambda_{1}^{j}(\xi, \eta), \ldots, \lambda_{v(j)}^{j}(\xi, \eta)\right\} \\
& \mathscr{P}_{j}(\xi, \eta)=\left\{P_{1}^{j}(\xi, \eta), \ldots, P_{v(j)}^{j}(\xi, \eta)\right\} \\
& \quad j=1, \ldots, J, \quad 1 \leqq J \leqq M, \quad M=\sum_{j=1}^{J} v(j),
\end{aligned}
$$


where

(i) $P_{i}^{j}(\xi, \eta), \quad i=1, \ldots, v(j), \quad j=1, \ldots, J$, are the associated eigenprojections to the eigenvalues $\lambda_{i}^{j}(\xi, \eta)$ and satisfy the relations (3.8).

(ii) for initial fixed point $\left(\xi_{f}, \eta_{f}\right) \in S^{1}-E, \lambda_{i}^{j}(\xi, \eta)\left(P_{i}^{j}(\xi, \eta)\right)$ agrees with $\lambda_{i+1}^{j}\left(\xi_{f}, \eta_{f}\right)\left(P_{i+1}^{j}\left(\xi_{f}, \eta_{f}\right)\right)$ after one time rotation with positive direction along $S^{1}$ in $S_{\boldsymbol{C}}^{1}(i=1, \ldots, v(j)-1: j=1, \ldots, J)$ and $\lambda_{v(j)}^{j}(\xi, \eta)\left(P_{v(j)}^{j}(\xi, \eta)\right)$ agrees with $\lambda_{1}^{j}\left(\xi_{f}, \eta_{f}\right)\left(P_{1}^{j}\left(\xi_{f}, \eta_{f}\right)\right)$ after one time rotation with positive direction along $S^{1}$ in $S_{\boldsymbol{C}}^{1}$. $(j=1, \ldots, J)$.

(iii) the elements of $\Lambda_{j}(\xi, \eta)$ are roots of the same irreducible polynomial of $Q(\lambda, \xi, \eta)$.

Proof. This lemma follows from the Lemma 3.1, the compactness of $S^{1}$ and analytic continuation along $S^{1}$.

Q.E.D.

Theorem 3.4. Assume the Condition I. Then, there exists the singular integral operator whose symbol has the properties (3.1).

Proof. Since the eigenprojections $P_{j}(\xi, \eta)$ associated with $\lambda_{j}(\xi, \eta)$, $j=1, \ldots, M$, have the $\operatorname{rank} m(j)$ in a neighborhood $U_{\xi_{0}, \eta_{0}}\left(\subset S_{\boldsymbol{C}}^{1}\right)$ of $\left(\xi_{\circ}, \eta_{\circ}\right) \in S^{1}$ in $\boldsymbol{C}^{2}$, there exist $m(j)$ linear independent row vectors $w_{1}^{j}(\xi, \eta), \ldots, w_{m(j)}^{j}(\xi, \eta)$ of $P_{j}(\xi, \eta)(j=1, \ldots, M)$. Put

$$
N_{\xi_{\circ}, \eta_{\circ}}(\xi, \eta)=\left(\begin{array}{c}
w_{1}^{1}(\xi, \eta) \\
\vdots \\
w_{m(1)}^{1}(\xi, \eta) \\
\vdots \\
w_{m(M)}^{M}(\xi, \eta)
\end{array}\right)
$$

Then, by the relations (3.8), we have that $N_{\xi_{0}, \eta_{0}}(\xi, \eta)$ is a non-singular matrix in $U_{\xi_{0}, \eta_{0}}$. By the analytic continuation, we extend $N_{\xi_{0}, \eta_{0}}(\xi, \eta)$ to the region $S_{\boldsymbol{C}}^{1}$. We retain for the function so obtained the same notation. Put $S_{\xi_{\circ}, \eta_{0}}(\xi, \eta)=N_{\xi_{\circ}, \eta_{\circ}}(\xi, \eta)^{*} N_{\xi, \diamond \eta_{\circ}}(\xi, \eta)$. Let $v$ be the least common multiple of numbers $v(1), \ldots, v(J)$. By the method of construction and Lemma 3.3, the function $N_{\xi_{0}, \eta_{0}}(\xi, \eta)$ has the period $v$. For any fixed $(\xi, \eta) \in S_{\overparen{C}}^{1} N_{\xi_{0}, \eta_{0}}(\xi, \eta)$ and $S_{\xi_{0}, \eta_{\circ}}(\xi, \eta)$ have respectively $v$ values $\left\{N_{\xi_{0}, \eta_{\circ}}^{1}\right.$ 
$(\xi, \eta), \ldots$, any fixed $\left.(\xi, \eta) \in S_{\boldsymbol{C}}^{1}, N_{\xi_{0}, \eta_{0}}^{v}(\xi, \eta)\right\}$, and $\left\{S_{\xi_{0}, \eta_{0}}^{1}(\xi, \eta), \ldots, S_{\xi_{0}, \eta_{0}}^{v}(\xi, \eta)\right\}$. Then $S_{\xi_{0}, \eta_{0}}^{i}(\xi, \eta)^{*}=S_{\xi_{0}, \eta_{0}}^{i}(\xi, \eta) i=1, \ldots, v$. By the method of construction and the theorem of identity, $N_{\xi_{\circ}, \eta_{0}}(\xi, \eta)$ is a non-singular matrix in a dense set $S_{d}$ of $S_{C}^{1}$ whose restriction on $S^{1}$ is dense in $S^{1}$. Next, we shall prove that $S_{\xi_{0}, \eta_{0}}^{i}(\xi, \eta) \cdot R(\xi, \eta)=R(\xi, \eta)^{*} \cdot S_{\xi_{0}, \eta_{0}}^{i}(\xi, \eta)$ where $(\xi, \eta) \in S^{1}$ $(i=1, \ldots, v)$. We assume that $N_{\xi_{0}, \eta_{0}}^{i}(\xi, \eta)$ is non-singular at $(\stackrel{\circ}{\xi}, \stackrel{\circ}{\eta}) \in S^{1}$. By the Condition I and the method of construction, $D=N_{\xi_{0}, \eta_{0}}^{i}(\stackrel{\circ}{\xi}, \stackrel{\circ}{\eta})$. $R(\stackrel{\circ}{\xi}, \stackrel{\circ}{\eta}) \cdot N_{\xi_{0}, \eta_{0}}^{i}(\stackrel{\circ}{\xi}, \stackrel{\circ}{\eta})^{-1}$ is a real diagonal matrix. Therefore, we have

$$
\begin{aligned}
& S_{\xi_{0}, \eta_{0}}^{i}(\stackrel{\circ}{\xi}, \stackrel{\circ}{\eta}) \cdot R(\stackrel{\circ}{\xi}, \stackrel{\circ}{\eta}) \\
&=N_{\xi_{0}, \eta_{0}}^{i}(\stackrel{\circ}{\xi}, \stackrel{\circ}{\eta})^{*} \cdot N_{\xi_{\circ} \eta_{0}}^{i}(\stackrel{\circ}{\xi}, \stackrel{\circ}{\eta}) \cdot N_{\xi_{0}, \eta_{0}}^{i}(\stackrel{\circ}{\xi}, \stackrel{\circ}{\eta})^{-1} \\
& \times D \cdot N_{\xi_{0}, \eta_{0}}^{i}(\stackrel{\circ}{\xi}, \stackrel{\circ}{\eta}) \\
&= N_{\xi_{0}, \eta_{0}}^{i}(\stackrel{\circ}{\xi}, \stackrel{\circ}{\eta})^{*} \cdot D \cdot N_{\xi_{0}, \eta_{0}}^{i}(\stackrel{\circ}{\xi}, \stackrel{\circ}{\eta}) \\
&=\left(D \cdot N_{\xi_{0}, \eta_{0}}^{i}(\stackrel{\circ}{\xi}, \stackrel{\circ}{\eta})\right)^{*} \cdot N_{\xi_{0}, \eta_{0}}^{i}(\stackrel{\circ}{\xi}, \stackrel{\circ}{\eta}) \\
&=\left(N_{\xi \xi_{0}, \eta_{0}}^{i}(\stackrel{\circ}{\xi}, \stackrel{\circ}{\eta}) \cdot R(\stackrel{\circ}{\xi}, \stackrel{\circ}{\eta})\right)^{*} \cdot N_{\xi_{0}, \eta_{0}}^{i}(\stackrel{\circ}{\xi}, \stackrel{\circ}{\eta}) \\
&= R(\stackrel{\circ}{\xi}, \stackrel{\circ}{\eta})^{*} \cdot S_{\xi_{0}, \eta_{0}}^{i}(\stackrel{\circ}{\xi}, \stackrel{\circ}{\eta}),
\end{aligned}
$$

where $S_{\xi_{0}, \eta_{0}}^{i}(\xi, \eta)$ corresponds to $N_{\xi_{0}, \eta_{0}}^{i}(\xi, \eta)$.

Since the set of non-singular points of $N_{\xi_{0}, \eta_{0}}(\xi, \eta)$ is dense in $S^{1}$, $S_{\xi_{0}, \eta_{0}}^{i}(\xi, \eta) \cdot R(\xi, \eta)=R(\xi, \eta)^{* \cdot} S_{\xi_{0}, \eta_{0}}^{i}(\xi, \eta), i=1, \ldots, v$ hold on $S^{1}$ by continuity. Put $\tilde{S}_{\xi_{0}, \eta_{0}}(\xi, \eta)=\sum_{i=1}^{v} S_{\xi_{0}, \eta_{0}}^{i}(\xi, \eta)$. Then, $\tilde{S}_{\xi_{0}, \eta_{0}}(\xi, \eta)$ is single valued analytic function in $S_{\boldsymbol{C}}^{1}$, a symmetric matrix with homogeneous degree zero in $(\xi, \eta)$ and positive definite in a open set $V_{\xi_{0}, \eta_{\circ}}$ such that $V_{\xi_{\circ}, \eta_{\circ}} \ni$ $\left(\xi_{0}, \eta_{0}\right)$ and $V_{\xi_{0}, \eta_{0}} \subset U_{\xi_{0}, \eta_{0}}$. Since $R(\xi, 0)=A \xi, S_{\xi_{0}, \eta_{0}}(\xi, 0)$ is a constant symmetric matrix, and $\tilde{S}_{\xi_{0}, \eta_{0}}(\xi, \eta) \cdot R(\xi, \eta)=R(\xi, \eta)^{*} \cdot \tilde{S}_{\xi_{0}, \eta_{0}}(\xi, \eta)$ on $S^{1}$. Thus, we can construct $\tilde{S}_{\xi_{0}, \eta_{0}}(\xi, \eta)$ at any $\left(\xi_{0}, \eta_{0}\right) \in S^{1}$. $S^{1}$ is compact. So, there exist finite points $\left(\xi_{1}, \eta_{1}\right), \ldots,\left(\xi_{e}, \eta_{e}\right)$ of $S^{1}$ such that $S_{1} \subset$ $\bigcup_{i=1}^{e} V_{\xi_{i}, \eta_{i}}$ and $S(\xi, \eta)=\sum_{i=1}^{e} \tilde{S}_{\xi_{i}, \eta_{i}}(\xi, \eta)$ is positive definite on $S^{1}$. Then, $S(\xi, \eta)$ is the symbol of the singular integral operator which we want 
to construct. Already, we proved the properties (i), (ii) and (iii) in (3.1). Next, we shall prove the property (iv). In [9], we proved that the eigenprojections $P_{h}(\kappa)\left(\widetilde{P}_{h}\left(\kappa^{\prime}\right)\right), h=1, \ldots, M$, of $T(\kappa)=A \kappa+B\left(T\left(\kappa^{\prime}\right)=\right.$ $\left.A+B \kappa^{\prime}\right)$ are analytic functions of $\kappa\left(\kappa^{\prime}\right)$ when $\kappa\left(\kappa^{\prime}\right)$ is real (real). Also, the eigenprojections of $T(\kappa)=\kappa\left(A+B \kappa^{-1}\right)$ coincide with those of $A+B \kappa^{-1}$. Therefore, $P_{h}(\kappa), h=1, \ldots, M$, are analytic in $\kappa$ when $\kappa$ is real and $\kappa=\infty$. So, $P_{h}(\kappa) \quad\left(\xi / \eta=\kappa, \eta \in \mathbb{R}^{1}-\{0\}\right), h=1, . ., M$, are analytic in $\kappa$ where $\kappa \in D=\left\{\kappa \in C^{1}|| \operatorname{Im} \kappa \mid<2 \delta_{1}\right\}, \quad \delta_{1}$ is a positive constant. Therefore, $S(\xi, \eta)$ has the property (iv).

Q.E.D.

\section{Energy Inequalities}

Since our method of proof is the same which Kajitani used in [4], we prove only the lemmas which are different from Kajitani's paper by reason of strong hyperbolicity.

Put $M(\tau, \eta)=A^{-1}(\tau I-i \eta B), \tau=\mu+i \sigma, \mu \geqq 0, \sigma \in \boldsymbol{R}^{1}, \eta \in \mathbb{R}^{1}$.

Lemma 4.1. Assume the Conditions $I$ and II. Fix $(\tau, \eta)=\left(i \sigma_{0}\right.$, $\left.\eta_{0}\right) \neq(0,0)$. Then, there exist positive numbers $r_{1}, r_{2}$ and a matrix $T(\tau, \eta)$ such that

(a) $T(\tau, \eta)$ is analytic in $U=\left\{(\tau, \eta)|| \tau-i \sigma_{0}\left|<r_{1},\right| \eta-\eta_{0} \mid<r_{2}\right\}$ and non-singular in $U$.

(b) $M(\tau, \eta)=T(\tau, \eta) M(\tau, \eta) T^{-1}(\tau, \eta)$ has the form

$$
M(\tau, \eta)=\left(\begin{array}{cccc}
M_{0}(\tau, \eta) & & 0 \\
& \ddots & \\
& & \ddots & \\
0 & & & M_{q}(\tau, \eta)
\end{array}\right)
$$

where

(i) $M_{j}, j=0, \ldots, q$, have $l_{j} \times l_{j}$ matrix.

(ii) the eigenvalues $\lambda$ of $M_{0}(\tau, \eta)$ satisfy

$$
|\operatorname{Re} \lambda|>C>0 .
$$

(iii) $M_{j}(\tau, \eta), j=1, \ldots, q$, have the form 


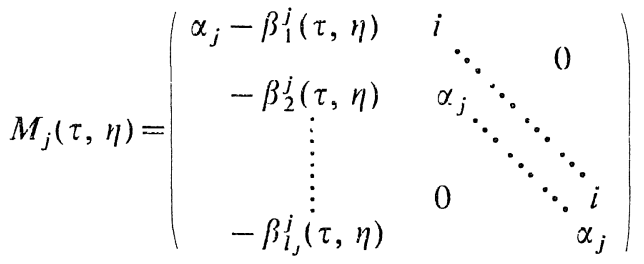

$$
\begin{aligned}
& \alpha_{j} \text { is pure imaginary and } \beta_{i}^{j}\left(i \sigma_{0}, \eta_{0}\right)=0\left(i=1, \ldots, l_{j}\right) \text {. }
\end{aligned}
$$

This lemma follows from Lemma 3.1 and Agranovich [1].

Remark 3. Lemma 4.1 is the same form for strictly hyperbolic systems [5].

We consider the boundary value problem with a parameter $\tau=$ $\mu+i \sigma, \mu>0$ :

$$
\left\{\begin{array}{lll}
(\tau-L) w=f & \text { in } & \boldsymbol{R}_{+}^{2} \\
\left.K \cdot w\right|_{x=0}=h & \text { in } & \mathbb{R}^{1} .
\end{array}\right.
$$

Lemma 4.2. Assume the Conditions I, II and III. Let $w(x, y)$ be any solution of (4.3) which belongs to $H^{1}\left(\mathbb{R}_{+}^{2}\right)$. Then, there exist positive constants $C_{1}$ and $\mu_{1}$ such that

$$
\begin{gathered}
\mu\|w\|^{2}+<w(0, \cdot)>2 \leqq C_{1}\left(\frac{1}{\mu}\|f\|^{2}+<h>^{2}\right) \\
\text { for any } \tau \text { with } \operatorname{Re} \tau \geqq \mu_{1} .
\end{gathered}
$$

Also, this lemma follows from Lemma 3.1 and Agranovich [1].

Lemma 4.3. Assume the Conditions $I$ and II. Then, there exists a positive constant $\gamma$ such that

(i) for $|\tau| \geqq \gamma|\eta|$, there exists an smooth matrix $H(\tau, \eta)$ such that $H(\tau, \eta) \cdot M(\tau, \eta) \cdot H^{-1}(\tau, \eta)$ is a diagonal matrix in $D=\{(\tau, \eta) \mid$ $|\tau| \geqq 2 \gamma|\eta|\}$ and the eigenvalues $\lambda_{j}(\tau, \eta)(j=1, \ldots, N)$ of $M(\tau, \eta)$ satisfy

$$
\left|\operatorname{Re} \lambda_{j}(\tau, \eta)\right| \leqq \text { const. } \mu \text { in } D \text {. }
$$

(ii) for $|\tau| \leqq 3 \gamma|\eta|$, there exists a $m=m(\tau, \eta)$ in the range 


$$
\frac{\delta_{1}}{2}|\eta| \leqq m \leqq \delta_{1}|\eta|
$$

such that

$$
\left|(\tau I-i(A \xi+B \eta))^{-1}\right| \leqq \frac{\text { const }}{|\check{\zeta}|}
$$

for $\xi$ with $\operatorname{Im} \xi=-m(\tau, \eta)$ where $\delta_{1}$ is a constant of (iv) in Theorem 3.4.

Proof. Let $\quad \tau \neq 0 . \quad M(\tau, \eta)=\frac{\tau}{i} A^{-1}\left(i I+\frac{\eta}{\tau} B\right) . \quad$ Also, $\quad M(\tau, 0)=\tau A^{-1}$ is diagonalizable by the Condition I. So, we apply Lemma 4.1 to $M(\tau$, $\eta$ ) at $(\tau, \eta)=(i, 0)$ and obtain (i). (ii) follows from Friedrichs and Lax [2] or Kajitani [3].

Q.E.D.

By the above arguments and the results of [4], we get the Main Theorem.

\section{References}

[1] Agranovich, M.S., Theorem on matrices depending on parameter and its applications to hyperbolic systems, Functional. Anal. i. Prilozen., 6 (1972), 85-93.

[2] Friedrichs, K. O. and Lax, P. D., Boundary value problems for first order operators, Comm. Pure Appl. Math., 18 (1965), 355-388.

[3] Kajitani, K., Initial boundary value problem for first order hyperbolic system, Publ. RIMS, Kyoto Univ., 7 (1971/72), 181-204.

[4] Kajitani, K. First order hyperbolic mixed problems, J. Math. Kyoto Univ., 11 (1971), 449-484.

[5] Ralston, J. V., Note on a paper of Kreiss, Comm. Pure Appl. Math., 24 (1971), 759-762.

[6] Rauch, J., Energy inequalities for hyperbolic initial boundary value problems, Thesis, New York Univ., (1971).

[7] Rauch, J., $\mathscr{L}^{2}$ is a continuable initial condition for Kreiss mixed problems, Comm, Pure Appl. Math., 25 (1972), 265-285.

[8] Strang, G., On strong hyperbolicity, J. Math. Kyoto Univ., 6 (1967), 397-417.

[9] Taniguchi, M., Mixed problem for hyperbolic systems of first order, Publ. RIMS Kyoto Univ., 8 (1972/73), 471-481. 